\title{
RESEARCH ON EVALUATION METHOD OF AIRBORNE LIDAR POSITIONING ACCURACY
}

\author{
ZHANG Yue $^{1,2}$,ZHOU Guoqing ${ }^{1,2}$,TANG Shihua ${ }^{1,2, ~ *}$, XING Pengwei ${ }^{1,2}$, HUANG Changcheng ${ }^{1,2}$ \\ ${ }^{1}$ College of Geomatics and Geoinformation, Guilin University of Technology, Guilin 54100,china, zhangyue.901@foxmail.com \\ ${ }^{2}$ Guangxi Key Laboratory of Spatial Information and Geomatics, Guilin 54100,china, (gzhou,yuetao)@glut.edu.cn
}

\section{Commission III, WG III/5}

KEY WORDS: Airborne LIDA, Accuracy Evaluation, Simulation Calculation, Evaluation Model, Circumscribed Circle Fitting

\begin{abstract}
:
Due to the semi-random characteristics of ground points collected by airborne LIDAR system, it is difficult to control the laser pin points to fall on the control points with known coordinates in actual measurement, so the accuracy can not be evaluated by directly comparing coordinate data. In this paper, based on the target plate designed by air-to-ground, the fuzzy c-means clustering analysis algorithm is proposed to extract the point cloud data on the target according to the different echo intensity data of laser on different ground objects. The center point coordinates of the target were fitted by the point cloud data on the target using the method of circumscribed circle of edge points, so as to realize the plane precision and elevation accuracy of airborne LIDAR system of evaluation. The results show that the model fitting method can quickly and effectively evaluate the accuracy of airborne LIDAR, and the method is simple and feasible.
\end{abstract}

\footnotetext{
* Corresponding author: TANG Shihua, E-mail: 528136765@qq.com
} 


\section{INSTRUCTIONS}

Airborne LIDAR (Light Detecting and held) is a kind of directly on the positioning of the new measurement technology(ZHANG xiaohong,2007). It originated from the traditional engineering measurement of laser ranging technology, through the laser range finder, attitude measurement device, D-GPS and remote sensing camera and other technical products integrated to directly obtain three-dimensional information on the ground, is the traditional radar technology and modern laser technology combined products. At present, LIDAR products have been launched by many manufacturers around the world, such as AHAB series scanning system of leica company, SHOALS system of Canada, CZMIL system of Optech company, and relatively mature technologies such as BEIKE and digital green earth in China(WANG Yue,2014.)(Qin Haiming, et al,2016)(YANG Xing-yu, et al,2016). In 2018, our scientific research team was awarded a guangxi innovative development project. The purpose of the project was to develop a set of airborne laser high-precision three-dimensional seabed measurement instrument, the structure principle of which is shown in figure 1 . As there are no relevant product standards and testing standards, the evaluation of the accuracy of lidar point cloud data is the key work in the testing and acceptance process.

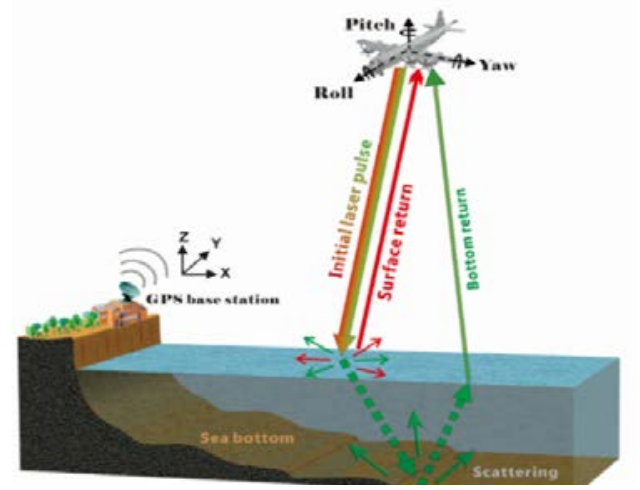

figure 1 Airborne lidar schematic diagram

Theoretically, the 3d coordinates of ground points obtained by airborne LIDAR directly do not need point position correction, matching and other processing work. However, in actual measurement, the placement error between each component of the system, the error of ranging and measuring Angle as well as the INU and DGPS measurement errors will affect the final point cloud accuracy(WANG Wei-wei, et al,2016). For the evaluation of laser radar, laser foot point points are usually plane precision and elevation accuracy evaluation(WANG Liying,2013.), by both the elevation accuracy evaluation is relatively simple, the most direct assessment method is the laser spot around the measured point elevation interpolation, by comparing the elevation in interpolation and elevation of the measured points can evaluate its elevation accuracy and evaluation results more reliable. It is usually impossible to directly evaluate the plane accuracy. In literature (WANG Shengyao,2013), a reflection mark higher than the ground is used for evaluation. Laser points on the mark are extracted according to the elevation information of the point cloud. In the book (ZHANG xiaohong,2007), a vertical wall is used for precision evaluation. This method requires the wall to be large enough with a large density of laser points. In the literature (WANG Shengyao,2013), it is also proposed to use the intensity information of laser echo to search for the same name points through echo data for accuracy evaluation. This method needs to make use of an effective algorithm to process the point cloud, and the accuracy depends on the density of the point cloud. According to the current research situation, there are many evaluation methods of airborne LIDAR for ground positioning accuracy, but there is no set of unified standards. The evaluation method is still in the process of research and improvement.

In this paper, based on the mathematical model of airborne lidar's ground positioning, the influence factors of errors in each part of the model are analyzed. According to the system parameters provided by the system developer, the positioning accuracy of the LIDAR system is simulated. Then, the target point cloud data extracted by fuzzy clustering analysis was fitted with the external tangent circle method to obtain the target center coordinates and compared with the target center coordinates accurately measured by the total station, and the plane root mean square error $\sigma_{x y}$ and elevation root mean square error $\sigma_{z}$ were calculated respectively as the model fitting accuracy(HUANG Xiaoyang, CHENG Shu, 2012).Finally, the simulation calculation precision and model fitting precision are compared and analysed.

\section{TPRECISION SIMULATION EVALUATION MODEL}

Airborne LIDAR is used to position the ground geometrically. The $3 d$ coordinates of the laser pin point in the scanner coordinate system are obtained by using the slant distance measured by the laser rangefinder and the Angle information measured by the POS system. After the transformation of inertial platform reference coordinate system and wgs-84 coordinate system, the point coordinate model(ZHANG xiaohong,2007), in GPS coordinate system can be obtained:

$$
\left[\begin{array}{l}
\mathrm{X}_{84} \\
Y_{84} \\
Z_{84}
\end{array}\right]=R_{\mathrm{w}} R_{\mathrm{G}} R_{\mathrm{N}}\left[R_{\mathrm{M}} R_{\mathrm{L}}\left[\begin{array}{l}
0 \\
0 \\
\rho
\end{array}\right]+\left[\begin{array}{c}
\Delta x_{I}^{L} \\
\Delta y_{I}^{L} \\
\Delta z_{I}^{L}
\end{array}\right]-\left[\begin{array}{c}
\Delta x_{I}^{G} \\
\Delta y_{I}^{G} \\
\Delta z_{I}^{G}
\end{array}\right]\right]+\left[\begin{array}{c}
\mathrm{x}_{0} \\
y_{0} \\
z_{0}
\end{array}\right]
$$

Where $\quad R_{\mathrm{N}}=$ The measured attitude angular rotation matrix.

$R_{\mathrm{N}}=$ The measured attitude angular rotation matrix.

$R_{\mathrm{M}}=$ The placement Angle rotation matrix;

$R_{\mathrm{L}}=$ Rotation matrix of scanning coordinate system;

$\rho=$ The distance measured by the laser rangefinder;

$\left[\begin{array}{lll}\mathrm{x}_{0} & \mathrm{y}_{0} & \mathrm{z}_{0}\end{array}\right]^{\mathrm{T}}=$ the coordinate of GPS phase center point. $R_{\mathrm{w}}$ and $R_{\mathrm{G}}$ are the transformation matrix of vertical and horizontal reference coordinates of and the current position respectively, which is regarded as the identity matrix in this paper. [ [ $\left.\begin{array}{lll}\Delta x_{I}^{L} & \Delta y_{I}^{L} & \Delta z_{I}^{L}\end{array}\right]^{\mathrm{T}}$ and $\left[\begin{array}{lll}\Delta x_{I}^{G} & \Delta y_{I}^{G} & \Delta z_{I}^{G}\end{array}\right]^{\mathrm{T}}$ are the offset between the laser launch center and the antenna phase center and the inertial platform respectively. This paper only focuses on the analysis of the influence of measuring Angle and ranging error on the accuracy of the foot points. For the convenience of research, equation (1) can be simplified and expressed as:

$$
P_{\mathrm{WGS}-84}=R_{\mathrm{N}}\left(R_{\mathrm{M}} S+\Delta P\right)+P_{0}
$$

In the formula, the attitude Angle rotation matrix is: $(\mathrm{H}, \mathrm{R}$ and $\mathrm{P}$ represent Heading, Roll and Pitch respectively)

$$
R_{\mathrm{N}}=R(H) * R(P) * R(R)=\left[\begin{array}{ccc}
\cos H \cos P & \cos H \sin P \sin R-\sin H \cos R & \sin H \sin R+\cos H \sin P \cos R \\
\sin H \cos P & \cos H \cos R+\sin H \sin P \sin R & \sin H \sin P \cos R-\cos H \sin R \\
-\sin P & \cos P \sin R & \cos P \cos R
\end{array}\right]
$$


Position error rotation matrix:

$$
R_{\mathrm{M}}=R(\Delta \gamma) * R(\Delta \alpha) * R(\Delta \beta)=\left[\begin{array}{ccc}
1 & -\Delta \gamma & \Delta \beta \\
\Delta \gamma & 1 & -\Delta \alpha \\
-\Delta \beta & \Delta \alpha & 1
\end{array}\right]
$$

In the formula $\Delta \gamma, \Delta \alpha, \Delta \beta$ respectively correspond to the placement Angle error of the XYZ triaxial, the placement Angle is a tiny Angle value, which generally needs to be measured in the air check.

Laser scanning coordinates in reference coordinates:

$$
S=\left[\begin{array}{ccc}
1 & 0 & 0 \\
0 & \cos \theta & -\sin \theta \\
0 & \sin \theta & \cos \theta
\end{array}\right]\left[\begin{array}{l}
0 \\
0 \\
\rho
\end{array}\right]=\left[\begin{array}{c}
0 \\
\rho \sin \theta \\
\rho \cos \theta
\end{array}\right] ; \quad P_{0}=\left[\begin{array}{l}
\mathrm{x}_{0} \\
y_{0} \\
z_{0}
\end{array}\right]
$$

Where $\quad \rho=$ The oblique distance

$\theta=$ The instantaneous scanning Angle.

$P_{0}=$ GPS antenna phase center coordinates

Offset of antenna phase center: $\Delta P=\left[\begin{array}{c}\Delta x_{I}^{L} \\ \Delta y_{I}^{L} \\ \Delta z_{I}^{L}\end{array}\right]-\left[\begin{array}{c}\Delta x_{I}^{G} \\ \Delta y_{I}^{G} \\ \Delta z_{I}^{G}\end{array}\right]=\left[\begin{array}{c}\Delta x \\ \Delta y \\ \Delta z\end{array}\right]$

Assuming that the errors are independent of each other, the partial derivatives of the parameters in the positioning model are calculated according to the measurement error propagation law, and the simulation accuracy expression of the laser pin point is obtained:

$$
\begin{aligned}
& m_{X}^{2}= \\
& \left(\frac{\partial X_{84}}{\partial H}\right)^{2} m_{H}^{2}+\left(\frac{\partial X_{84}}{\partial P}\right)^{2} m_{P}^{2}+\left(\frac{\partial X_{84}}{\partial R}\right)^{2} m_{R}^{2}+\left(\frac{\partial X_{84}}{\partial \rho}\right)^{2} m_{\rho}^{2}+\left(\frac{\partial X_{84}}{\partial \theta}\right)^{2} m_{\theta}^{2}+\left(\frac{\partial X_{84}}{\partial \Delta \gamma}\right)^{2} m_{\Delta \gamma}^{2}+\left(\frac{\partial X_{84}}{\partial \Delta \beta}\right)^{2} m_{\Delta \beta}^{2}+\quad\left(\frac{\partial X_{84}}{\partial \Delta \alpha}\right)^{2} m_{\Delta \alpha}^{2}+ \\
& \left(\frac{\partial X_{84}}{\partial \Delta x}\right)^{2} m_{\Delta x}^{2}+\left(\frac{\partial X_{84}}{\partial \Delta y}\right)^{2} m_{\Delta y}^{2}+\left(\frac{\partial X_{84}}{\partial \Delta z}\right)^{2} m_{\Delta z}^{2}+m_{X_{0}}^{2} \\
& m_{Y}^{2}= \\
& \left(\frac{\partial Y_{84}}{\partial H}\right)^{2} m_{H}^{2}+\left(\frac{\partial Y_{84}}{\partial P}\right)^{2} m_{P}^{2}+\left(\frac{\partial Y_{84}}{\partial R}\right)^{2} m_{R}^{2}+\left(\frac{\partial Y_{84}}{\partial \rho}\right)^{2} m_{\rho}^{2}+\left(\frac{\partial Y_{84}}{\partial \theta}\right)^{2} m_{\theta}^{2}+\left(\frac{\partial Y_{84}}{\partial \Delta \gamma}\right)^{2} m_{\Delta \gamma}^{2}+\left(\frac{\partial Y_{84}}{\partial \Delta \beta}\right)^{2} m_{\Delta \beta}^{2}+\quad\left(\frac{\partial Y_{84}}{\partial \Delta \alpha}\right)^{2} m_{\Delta \alpha}^{2}+ \\
& \left(\frac{\partial Y_{84}}{\partial \Delta x}\right)^{2} m_{\Delta x}^{2}+\left(\frac{\partial Y_{84}}{\partial \Delta y}\right)^{2} m_{\Delta y}^{2}+\left(\frac{\partial Y_{84}}{\partial \Delta z}\right)^{2} m_{\Delta z}^{2}+m_{Y_{0}}^{2} \\
& m_{Z}^{2}= \\
& \left(\frac{\partial Z_{84}}{\partial H}\right)^{2} m_{H}^{2}+\left(\frac{\partial Z_{84}}{\partial P}\right)^{2} m_{P}^{2}+\left(\frac{\partial Z_{84}}{\partial R}\right)^{2} m_{R}^{2}+\left(\frac{\partial Z_{84}}{\partial \rho}\right)^{2} m_{\rho}^{2}+\left(\frac{\partial Z_{84}}{\partial \theta}\right)^{2} m_{\theta}^{2}+\left(\frac{\partial Z_{84}}{\partial \Delta \gamma}\right)^{2} m_{\Delta \gamma}^{2}+\left(\frac{\partial Z_{84}}{\partial \Delta \beta}\right)^{2} m_{\Delta \beta}^{2}+\quad\left(\frac{\partial Z_{84}}{\partial \Delta \alpha}\right)^{2} m_{\Delta \alpha}^{2}+ \\
& \left(\frac{\partial Z_{84}}{\partial \Delta x}\right)^{2} m_{\Delta x}^{2}+\left(\frac{\partial Z_{84}}{\partial \Delta y}\right)^{2} m_{\Delta y}^{2}+\left(\frac{\partial Z_{84}}{\partial \Delta z}\right)^{2} m_{\Delta z}^{2}+m_{Z_{0}}^{2}
\end{aligned}
$$

Where:

$m_{H}^{2}, m_{P}^{2}, m_{R}^{2}=$ The errors in the three attitude Angle parameters corresponding to IMU respectively.

$m_{\rho}^{2}, m_{\theta}^{2}=$ The errors in the parameters of laser ranging and scanning Angle respectively.

$m_{\Delta \gamma}^{2}, m_{\Delta \beta}^{2}, m_{\Delta \alpha}^{2}=$ The errors in the three placement Angle parameters respectively;

$m_{\Delta x}^{2}, m_{\Delta y}^{2}, m_{\Delta z}^{2}=$ The errors in the eccentricity parameter respectively;

$m_{X_{0}}^{2}, m_{Y_{0}}^{2}, m_{Z_{0}}^{2}=$ The errors of GPS $3 d$ coordinate components respectively.

The specific expressions of the first partial derivatives of each variable have been derived in a large number of literatures. According to the simulation accuracy expression of laser pin point, the data of laser pin point near the center on the target can be extracted by manual extraction, and the simulation accuracy of the target center can be estimated by combining with the error source parameters of airborne LIDAR system. The system error parameters and their indexes under certain flight conditions are shown in table 1.

Table 1 Error sources and parameters

\begin{tabular}{ccc}
\hline Error types & Parameter & Mean Square Error \\
\hline \multirow{2}{*}{ Laser scanner } & $\rho$ & $0.005 \mathrm{~m}$ \\
& $\theta$ & $0.0028^{\circ}$ \\
\hline Attitude & $\mathrm{H}$ & $0.008^{\circ}$ \\
angle & $\mathrm{P}$ & $0.005^{\circ}$ \\
& $\mathrm{R}$ & $0.005^{\circ}$ \\
\hline \multirow{2}{*}{ Placement } & $\Delta \gamma$ & $0.01^{\circ}$ \\
angle & $\Delta \beta$ & $0.008^{\circ}$ \\
& $\Delta \alpha$ & $0.008^{\circ}$ \\
\hline Eccentric & $\Delta x$ & $0.001 \mathrm{~m}$
\end{tabular}

\begin{tabular}{ccc} 
distance & $\Delta y$ & $0.001 \mathrm{~m}$ \\
& $\Delta z$ & $0.001 \mathrm{~m}$ \\
\hline \multirow{3}{*}{ GPS } & $X_{0}$ & $0.05 \mathrm{~m}$ \\
& $Y_{0}$ & $0.05 \mathrm{~m}$ \\
& $Z_{0}$ & $0.10 \mathrm{~m}$ \\
\hline
\end{tabular}

The attitude Angle data obtained by INS system were calculated, and the average angles of pitch Angle, yawing Angle and side roll Angle were calculated as $\pm 3^{\circ}, \pm 4^{\circ}$ and $\pm 3^{\circ}$ respectively. When the scanning Angle was set as $\pm 30^{\circ}$ and the flight relative height was $80 \mathrm{~m}$, the maximum ranging was obtained as $92.38 \mathrm{~m}$. Combined with the previous analysis, the data in table 1 was substituted into formula 1 and formula 3 to calculate the simulation accuracy of $3 \mathrm{~d}$ coordinates of laser pin points. The results showed that the elevation simulation accuracy (error in RMS) was $0.119 \mathrm{~m}$, and the plane simulation accuracy was $\left(0.133^{2}+0.147^{2}\right)^{1 / 2}=0.198 \mathrm{~m}$.

\section{ACCURACY EVALUATION METHOD}

Airborne lidar obtains discrete three-dimensional laser point cloud data, and different lidar systems obtain different point cloud densities. Therefore, it is difficult to directly obtain the target center point coordinates through manual point finding like photogrammetric images, so it is difficult to directly evaluate the accuracy. In the experiment, a circular target board was set up in the calibration field. The coordinates of the center point of the target were accurately measured by the total station. The precision of plane coordinates was better than $5 \mathrm{~mm}$, and the precision of elevation was better than $2 \mathrm{~cm}$.In this paper, the point cloud containing the target part was manually segmented to obtain the point cloud containing the target part. The coordinates of the target center point were fitted with the method of outer tangent circle. The accuracy evaluation process was shown in flowchart 1 . 


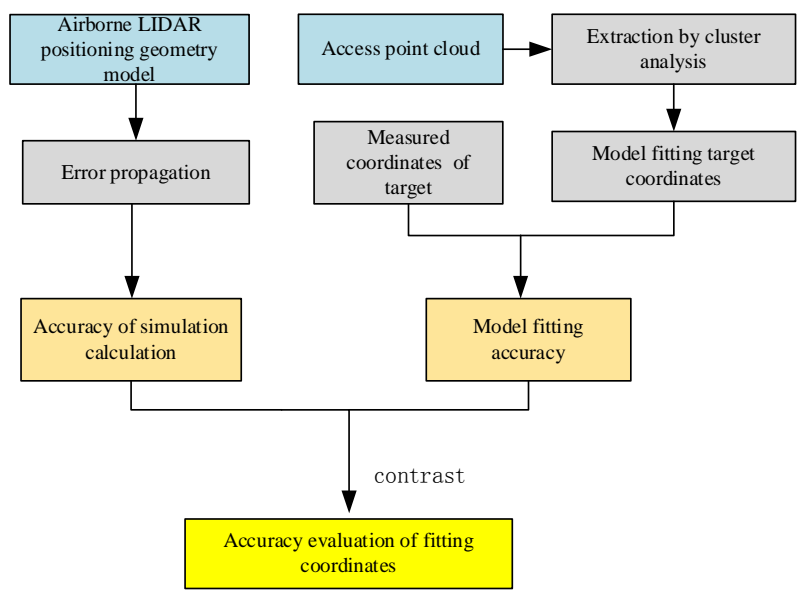

Flowchart 1 Accuracy Evaluation Process

\subsection{Target point cloud extraction}

In the experiment, the planar target is made of materials that have a strong reflection on the laser. The echo intensity data of the point cloud on the target is much larger than that of the surrounding point cloud, so the target point cloud can be accurately extracted from the scanning scene through the clustering analysis algorithm according to the reflection intensity information of the reflected light. Fuzzy c-mean clustering algorithm was used to classify point clouds, and the cluster number c was set to obtain point clouds with different types of reflection intensity. Then, high reflectivity data was extracted, and the target point cloud data was obtained after removing abnormal points. Its calculation principle is expressed as follows:

$$
\begin{gathered}
\mathrm{J}=\sum_{i=1}^{c} \sum_{j=1}^{N} u_{i j}^{m}\left\|x_{j}-v_{i}\right\|^{2} \\
v_{i}=\frac{\sum_{j=1}^{N} u_{i j}^{m} x_{j}}{\sum_{j=1}^{N} u_{i j}^{m}} \\
\sum_{k=1}^{c}\left(\frac{\left\|x_{j}-v_{i}\right\|}{\left\|x_{j}-v_{k}\right\|}\right)^{\frac{2}{m-1}}
\end{gathered}
$$

The reflectivity set $\mathrm{X}\left\{x_{1} \ldots, x_{j}, \ldots, x_{N}\right\}$ of $\mathrm{N}$ data points in the point cloud is classified into $\mathrm{C}$ class. In the formula, $u_{i j}$ represents the membership degree of sample $x_{j}$ to the i class, $u_{i j}$ $\in[0,1], \sum_{i=1}^{N} u_{i j}>0, \sum_{i=1}^{C} u_{i j}=1, v_{i}$ is the center of the i class, $\|\bullet\|$ means Euclidean distance, $m>1$ is fuzzy weights. The objective function $\mathrm{J}$ was optimized by means of iterative optimization. As the number of iterations increased, the clustering center of class i was updated accordingly.

Target point cloud extraction is mainly carried out in 2 steps:

1.Rough extraction of target point cloud data by cluster analysis method.

2.Calculate the mean $I_{\text {mean }}$ of the maximum and minimum light reflection intensity of the target point cloud obtained from rough extraction, take 1.5 times $I_{\text {mean }}$ as the threshold, and select the point cloud larger than the threshold as the final extraction result of the target point cloud.

\subsection{Target center location method}

At present, for the research on the positioning of target center, is the most commonly used method in average method respectively to calculate $3 \mathrm{~d}$ coordinates of each point on the target of average $\bar{X}_{i}, \bar{Y}_{i}, \bar{Z}_{i}$ as target center. Although this method can accurately calculate the target center coordinate, it requires the point cloud data on the target to be evenly and completely distributed, and the error of the target center coordinate calculated for the target point cloud with uneven sampling or partial incomplete will be relatively large. In this paper, an outer tangent circle method is designed to calculate the bull 's-eye coordinates. Its principle is shown in figure 2.Given the coordinates of 3 or more points on the circumference, the coordinates of the center of the circle can be fitted and calculated (ZHOU Xizheng, et al,2012), so the edge points of the target can be extracted first, and the coordinates of the center of the circle can be calculated and fitted.

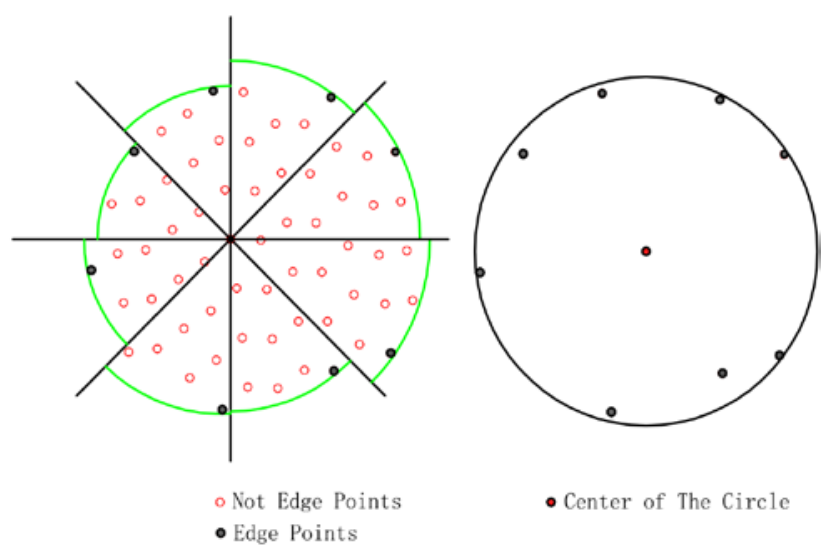

Figure 2 Circumscribed circle fitting

The main calculation steps of the target center are as follows:

(1) Select one point of the target point cloud as the center and record the coordinates of the point.

(2) Divide the point cloud into several fan-shaped regions according to a certain fixed Angle at the center point (for example, divide the point cloud into 8 fan-shaped regions according to $45^{\circ}$ in this paper), and calculate that the farthest point from the center point in each region is the edge point of the region.

(3) Extract the coordinates of the edge points of each area, and use the minEnclosingCircle() function in OpenCV to get the fitting center coordinates $\mathrm{X}_{i}, \mathrm{Y}_{i}$ and radius $\mathrm{R}_{i}$.

\subsection{Accuracy evaluation model}

After obtaining the central coordinates $\mathrm{X}_{i} 、 \mathrm{Y}_{i}$ of each target, and comparing them with the measured coordinates $x_{0 j}, y_{0 j}$, the RMS error of the plane can be calculated according to equation (7) to evaluate the plane accuracy of the Airborne LIDAR. Circumscribed circle fitting the target center only plane coordinates cannot elevation accuracy evaluation, so will each target point cloud on average elevation of $\bar{Z}_{i}$ compared with measured value $z_{0 j}$, according to the type (8) calculating the root mean square error of the elevation of $\sigma_{z}$ as index of vertical accuracy.

$$
\begin{gathered}
\sigma_{s}=\sqrt{\frac{\sum_{j=1}^{m}\left[\left(\mathrm{X}_{i}-x_{0 j}\right)^{2}+\left(\mathrm{Y}_{i}-y_{0 j}\right)^{2}\right]}{m}} \\
\sigma_{z}=\sqrt{\frac{\sum_{j=1}^{m}\left(\frac{\sum_{i=1}^{n} z_{i}}{n}-z_{0 j}\right)^{2}}{m}}
\end{gathered}
$$


In the formula:

$\mathrm{m}=$ The total number of targets.

$\mathrm{j}=$ The target serial number.

$\mathrm{n}=$ The quantity of laser points on the $\mathrm{j}$ target.

$\mathrm{i}=$ The serial number of laser points on the $\mathrm{j}$ target.

$\left(x_{0 j}, y_{0 j}, z_{0 j}\right)=$ The precise coordinate of the target center of the j block.

\section{EXPERIMENT AND CONCLUSION ANALYSIS}

\subsection{Assess site data acquisition}

The experimental data in this paper are the prototype test data provided by the research team of lidar system. The forest land with rich plane features in guangxi is taken as the experimental area, and the terrain in the area is flat and the landform is rich. In the test area, 6 targets were evenly distributed at equal intervals, with a target radius of $0.8 \mathrm{~m}$ and $0.5 \mathrm{~m}$ higher than the nearby ground, which was significantly different from the surrounding ground features, and could be well identified from the laser point cloud. The center of the target point position was accurately determined by the total station. The horizontal precision of the point position was better than $1 \mathrm{~cm}$, and the elevation precision was better than $2 \mathrm{~cm}$.Some point clouds in the test area are displayed according to the reflection intensity as shown in figure 3 .

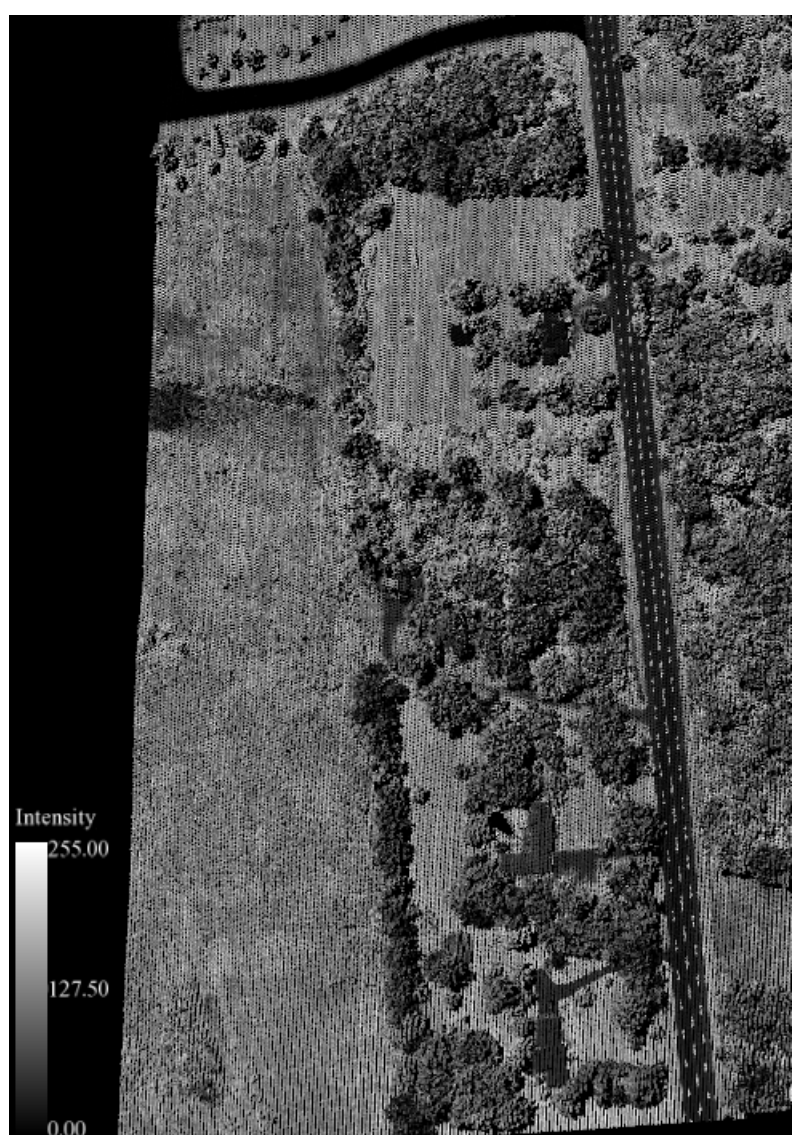

Fig.3 Point Cloud Data of the Experimental Area

\subsection{Target center positioning accuracy}

Select experiment zone 4 piece of point cloud distribution measurement targets were tested with better effect, extraction of target coordinate point cloud data on import Cass 9.0 software exhibition display and circumscribed circle fitting effect as shown in figure 4, Application of the method to calculate the target center coordinates and comparing with the results of the measured coordinates $\mathrm{X}$ and $\mathrm{Y}$ values, the residue situation as shown in table 2 .

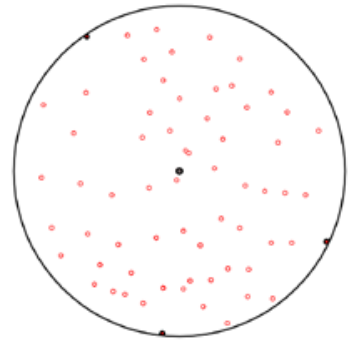

QZ01

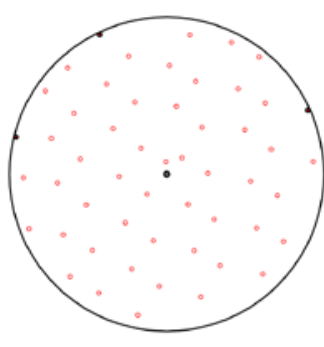

QZ04

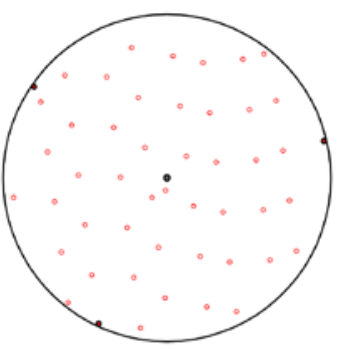

QZ03

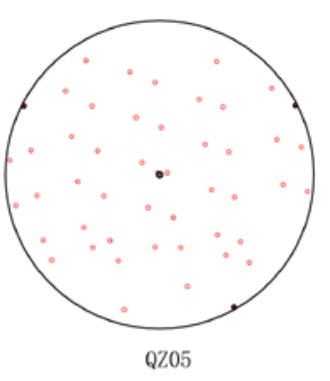

Figure 4 Shows the Extracted Target Point Cloud
Table 2 shows that the maximum residual in the $\mathrm{X}$ direction of the target is $13.8 \mathrm{~cm}$, and the maximum residual in the $\mathrm{Y}$ direction is $16.7 \mathrm{~cm}$. The precision of the fitting plane is better than $\left(13.8^{2}+16.7^{2}\right)^{1 / 2}=21.66 \mathrm{~cm}$. The accuracy is close to the simulation results, so it can be considered that the center coordinates of the target cloud can be calculated with high precision by using the circum-tangent circle fitting method for the uniformly distributed target cloud. 
Table 2 The fitting coordinate and the measured coordinate value

\begin{tabular}{ccccccc}
\hline Target & \multicolumn{2}{c}{ Fitting Coordinates } & \multicolumn{2}{c}{ Measured Coordinate } & \multicolumn{2}{c}{ Residual $(\mathrm{cm})$} \\
\cline { 2 - 7 } Number & $\mathrm{X}$ & $\mathrm{Y}$ & $\mathrm{X}$ & $\mathrm{Y}$ & $\Delta \mathrm{X}$ & $\Delta \mathrm{Y}$ \\
\hline QZ01 & $* * 630.550$ & $* * 069.980$ & $* * 630.622$ & $* * 069.851$ & -7.2 & 12.9 \\
QZ03 & $* * 631.858$ & $* * 042.675$ & $* * 631.763$ & $* * 042.532$ & 9.5 & 14.3 \\
QZ04 & $* * 632.925$ & $* * 027.757$ & $* * 632.787$ & $* * 027.924$ & 13.8 & -16.7 \\
QZ05 & $* * 634.063$ & $* * 012.571$ & $* * 634.194$ & $* * 012.482$ & -13.1 & 8.9 \\
\hline
\end{tabular}

\subsection{Accuracy evaluation results}

Integration errors of sensors, such as scanning system, IMU and GNSS of airborne lidar system, as well as errors caused by environmental factors; Under the flight conditions with a speed of $6 \mathrm{~m} / \mathrm{s}$ and an altitude of $80 \mathrm{~m}$, the calculated simulation calculation accuracy is compared with the evaluation result, as shown in table 3.

\section{CONCLUSION}

In this paper, the accuracy of ground target point cloud is simulated and calculated according to the mathematical model, and the peripheral tangent circle of the edge points is used to fit the target center for accuracy evaluation. The experimental results show that the precision of the target center coordinate fitted by this method is equivalent to that of simulation, and it is considered that this method can be used to evaluate the accuracy of airborne lidar positioning.

\section{ACKNOWLEDGEMENTS}

This paper is financially supported by the National Natural Science of China under Grant numbers 41431179,41961065; Guangxi Innovative Development Grand Grant under the grant number: GuikeAA18118038, GuikeAA18242048; the National Key Research and Development Program of China under Grant numbers 2016YFB0502501 and the BaGuiScholars program of Guangxi

\section{REFERENCES}

ZHANG xiaohong,2007: Airborne LIDAR measurement technology theory and method[M]. Wuhan University Press, 2007.

WANG Yue[1],2014. Current Status and Development of Airborne Laser Bathymetry Technology[J]. Journal of Geomatics, 2014(3)

Qin Haiming, Wang Cheng, Xi Xiaohuan, et al,2016. Development of Airborne Laser Bathymetric Technology and Applications[J]. Remote Sensing Technology and Application, 2016, Issue(4).

YANG Xing-yu, SU Jin-san, WANG Yuan-qing, et al,2016. Research the Development of International Laser Imaging Radar System[J]. Laser Journal, 2016, 37(1):1-4.

WANG Wei-wei, ZHANG Hui, et al,2016. Research of Calibration Site Design and Calibration Precision of Airborne
Table 3 Comparison of Accuracy

\begin{tabular}{cccc}
\hline System parameters & Simulation & Fitting \\
\hline Sweep frequency/Hz & \multicolumn{3}{c}{$5 \sim 20$} \\
Scan Angle $/\left({ }^{\circ}\right)$ & \multicolumn{3}{c}{ \pm 30} \\
Flying height $/ \mathrm{m}$ & \multicolumn{3}{c}{80} \\
Flying speed $/(\mathrm{m} / \mathrm{s})$ & & 6 & \\
Plane precision/cm & 19.8 & & 21.66 \\
Elevation precision/cm & 11.9 & & 10.2 \\
\hline
\end{tabular}

LiDAR[J]. Beijing Surveying and Mapping, 2016, No.129(4):89-92.

WANG Liying,2013. Theory and Methods of Error Processing for Airborne LIDAR Date[M]. Surveying and Mapping Press, 2013.

WANG Shengyao[1],2013. The Horizontal Accuracy Assessment of Airborne LiDAR Data[J]. Geomatics \& Spatial Information Technology, 2013, 36(7):54-56.

WU Xiping,2013. The theory of genetic algorithm optimization fuzzy clustering algorithm in image segmentation[J]. Electronic Test, 2013(3):154-155.

ZHOU Xizheng, LIU Chuanrui, et al,2012. Reliability Analysis of Multiple Points Coordinates on an Arc to Fitting Circle Center Position[J]. Site Investigation Science and Technology, 2012(4):54-57.

HUANG Xiaoyang, CHENG Shu, 2012.Accuracy of Airborne Laser Radar to Ground Positioning[J]. Journal of Geomatics, 2012, 37(4):10-12. 